\title{
A Maturity-Onset Diabetes of The Young (MODY) 5 Young Woman With HNF1B Heterozygosity Missing Based On 17q12 Recurrent Deletion Syndrome
}

\section{Ying Cheng ( $\square$ chengyingxgg@163.com )}

The General Hospital of Western Theater Command PLA https://orcid.org/0000-0002-5223-4939

\section{Da-Peng Zhong}

The General Hospital of Western Theater Command PLA

\section{Li Ren}

The General Hospital of Western Theater Command PLA

\section{Hang Yang}

The General Hospital of Western Theater Command PLA

\section{Chen-Fu Tian}

The General Hospital of Western Theater Command PLA

\section{Research Article}

Keywords: Maturity-onset diabetes of the young 5 (MODY 5), Hepatocyte nuclear factor 1 homeobox $b$ gene (HNF1B), 17q12 Recurrent deletion syndrome

Posted Date: October 27th, 2021

DOl: https://doi.org/10.21203/rs.3.rs-964266/v1

License: (c) (i) This work is licensed under a Creative Commons Attribution 4.0 International License.

Read Full License 


\title{
A maturity-onset diabetes of the young (MODY) 5 young woman with HNF1B heterozygosity missing based on $17 q 12$ recurrent deletion syndrome
}

\author{
Ying Cheng ${ }^{1}$, Da-Peng Zhong ${ }^{1}$, Li Ren ${ }^{1}$, Hang Yang ${ }^{1}$, Chen-Fu Tian ${ }^{1}$
}

*Correspondence: chengyingxgg@163.com

1Department of Endocrinology, The General Hospital of Western Theater Command PLA, No. 270 Rongdu Avenue, Jinniu District, Chengdu 610083, Sichuan Province, People's Republic of China

\begin{abstract}
Maturity-onset diabetes of the young type 5 (MODY5) is a rare subtype of MODYs. It caused by mutations of the hepatocyte nuclear factor 1 homeobox b gene (HNF1B). A 21-year-old young woman was admitted to our hospital for severe malnutrition and gastrointestinal symptoms. At age 20, she was diagnosed with type 2 diabetes mellitus and was administered with oral antidiabetic drugs. Soon afterwards, the patient discontinued the medication on her own accord, and then went to the hospital again due to diabetic ketoacidosis. After insulin treatment, diabetic ketoacidosis was cured and blood glucose was controlled satisfactorily. But intractable nausea, vomiting and persistent weight loss was stubborn. Further examination revealed that the patient had hypokalemia and hard rectification hypomagnesemia. Genetic testing revealed about $1.85 \mathrm{Mb}$ heterozygous fragment deletion on chromosome 17 and deletion of exons 1-9 of HNF1B heterozygosity missing was approved. Finally, the patient was diagnosed MODY 5 with HNF1B heterozygosity missing based on 17q12 recurrent deletion syndrome.
\end{abstract}

Key words: Maturity-onset diabetes of the young 5 (MODY5), Hepatocyte nuclear factor 1 homeobox b gene (HNF1B), 17q12 Recurrent deletion syndrome

\section{Introduction}

Maturity-onset diabetes of the young (MODY) is the most common type of monogenic diabetes. To the best of our knowledge, 14 candidate genes are involved in the pathogenesis of MODY ${ }^{[1,2]}$. Mutations in the hepatocyte nuclear transcription factor 1 homeobox $\alpha$ (HNF 1A), glucokinase gene (GCK) and hepato-nuclear factor 4 homeobox (HNF 4A) are the commonest subtype ${ }^{[3]}$. MODY 5 is caused by mutation of the transcription factor HNF1B gene and is a rare subgroup of MODYs. It accounts about for about 2-6\% patients with MODYs. Although most MODY 5 is caused by HNF1B point mutations, we herein discuss a case who suffered from HNF1B deletion due to $17 \mathrm{q} 12$ recurrent deletion syndrome.

\section{Case presentation}

A 21-year-old young woman with diabetes was admitted to our hospital due to recurrent nausea and vomiting. She experienced polydipsia and polyuria a year ago and was diagnosed with diabetes mellitus. Initially she was treated with acarbose and blood glucose was not monitored. After 6 months, she discontinued antidiabetic drug at her own discretion. About two months ago, she was taken to the hospital in a coma and was diagnosed with diabetic ketoacidosis. Since then, insulin has been used to control blood glucose. Even though blood glucose control was up to standard, she still suffered from serious nausea, vomiting, 
abdominal pain and fatigue. Her body weight gradually lost $15 \mathrm{~kg}$ in two months. In order to improve these symptoms, the patient was admitted to our hospital. This patient's maternal grandfather had been diagnosed with type 2 diabetes mellitus, but her parents are in good health. There was no other family history.

On admission, the patient was in a status of very cachectic nutritional. She was $161.0 \mathrm{~cm}$ tall and weighs only $35.9 \mathrm{~kg}$. Body mass index (BMI) was $13.85 \mathrm{~kg} / \mathrm{m}^{2}$. Blood pressure was 111 vs. $54 \mathrm{mmHg}$. The patient has got serious malnutrition and muscular dystrophy. The physical examination of the heart and lungs showed no abnormalities. The patient complained of persistent lower abdominal pain, but the physical examination showed no abnormal abdominal signs.

After hospital admission, insulin was sustained to be used and blood glucose was well controlled. Both enteral and parenteral nutrition were given to improve nutritional status. After hospital admission, a series of examinations were performed to find the cause of nausea, vomiting, and weakness. At the same time, the etiology of the patient's diabetes was sought.

The patient's liver and kidney function were normal. Glycosylated hemoglobin (HbA1c) was 6.7\%. Fasting serum insulin was $4.12 \mathrm{mU} / \mathrm{L}$ and fasting serum c-peptide was $0.39 \mathrm{ng} / \mathrm{ml}$. Type 1 diabetes mellitus was excluded because of negative diabetes autoantibodies, e.g. glutamate decarboxylase antibody (GADA), protein tyrosine phosphatase antibody (IA-2A), insulin antibody (IAA) and islet cell antibody (ICA). Hypomagnesemia and hypokalemia were found. Serum magnesium was $0.37 \mathrm{mmol} / \mathrm{L}$ and the fractional magnesium excretion (FEMg) was $9.7 \%$. Serum potassium was $3.32 \mathrm{mmol} / \mathrm{L}$ and urine potassium/creatinine ratio was $5.93 \mathrm{mmol} / \mathrm{mmol}$. The patient's thyroid, parathyroid, pituitary, and adrenal functions were normal. Abdominal ultrasonography revealed intrahepatic calcification. Biliary system, pancreas, spleen and genitourinary system were normal. Gastroscopic examination revealed non-atrophic gastritis with bile reflux.

Hypokalemia was quickly corrected after potassium chloride supplementation, but hypomagnesemia was refractory. After obtained informed consent from the patient, genetic testing was adopted. By the full exon detection method of chip capture high-throughput sequencing, there was a large heterozygous fragment deletion on chromosome 17, the deletion interval was chr17:34493374 to 36347081 , about $1.85 \mathrm{Mb}$. And deletion of exons 1-9 of HNF1B heterozygosity missing was approved (Fig.1).

Ultimately, maturity-onset diabetes of the young (MODY) 5 with HNF1B heterozygosity missing based on $17 \mathrm{q} 12$ recurrent deletion syndrome was diagnosed. 


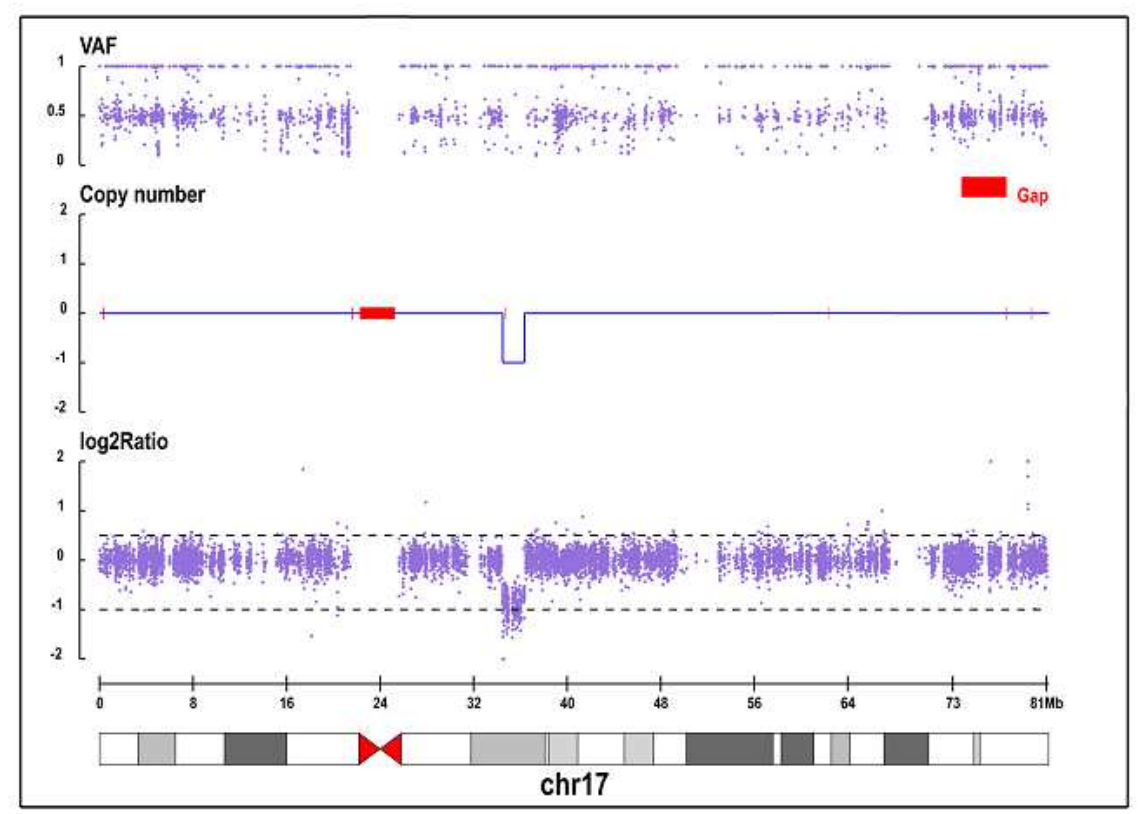

Fig. 1 Microarray profile of chromosome 17 showing the deleted region

\section{Discussion}

MODY is an autosomal dominant type of monogenic diabetes. It is caused by mutations in one of the genes that resulted in $\beta$-cell dysfunction ${ }^{[4]}$. The refined diagnostic criteria for MODY include: persistent hyperglycemia in early adulthood (typically before 30 years), clinical features incompatible with type 1 or type 2 diabetes mellitus (T1DM, T2DM), diabetes in at least one first-degree relative, evidence of residual pancreatic function, and absence of beta cell autoimmunity ${ }^{[1]}$. This patient met four criteria out of five standards. And none of her first-degree relatives were diagnosed with diabetes. But a second-degree relative was diagnosed with diabetes mellitus.

Although MODY is a most common monogenetic inherited diabetes, diagnosis is often delayed due to insidious onset of symptoms, lack of an obvious family history of diabetes and overlap of clinical features with T1DM and T2DM. Particularly accurate etiological diagnosis is challenging. Despite many biomarkers such as complement components, high-sensitivity C-reactive protein have been tested as auxiliary diagnostic tools, a definitive diagnosis depends on genetic testing ${ }^{[5]}$. This patient was initially diagnosed with type 2 diabetes and oral hypoglycemic medication was administered to control blood glucose. However, one year after the diagnosis of diabetes, diabetic ketoacidosis occurred and the function of pancreatic islet cell was failure. Although her maternal grandfather was diagnosed with type 2 diabetes mellitus, there were no first-degree relatives with diabetes. Therefore, the diagnosis of MODY was delayed.

Up to now, fourteen subtypes of MODY have been reported ${ }^{[4]}$. There is no concise or standardized diagnostic algorithm for MODY. However, some clinical signs indicate that the patient may have inherited diabetes. For example, there was hyperinsulinism during infancy, low triglyceride level, pancreatic agenesis, renal anomalies, genital anomalies and so on. This patient had serious fatigue, rapid weight loss, and refractory hypomagnesemia even after blood glucose was controlled. All these clinical characteristics suggest a specific type of 
diabetes. Genetic tests confirmed that the patient was MODY5 due to deletion of HNF1B gene.

Although MODY5 is inherited in an autosomal dominant pattern, genetic abnormalities can also occur spontaneously. It has been suggested that the new occurrence rate of HNF1B gene deletion can be as high as $50 \%$. MODY5 is often one of the manifestations of 17q12 recurrent deletion syndrome.

$17 \mathrm{q} 12$ recurrent deletion syndrome is also known as renal cysts and diabetes (RCAD) syndrome ${ }^{[6]}$. It is a distinct, recurrent chromosomal aberration. The aberration encompass multiple genes, HNF1B, LHX1, and ACACA, among others. Deletion of HNF1B is associated with MODY5. HNF1B is expressed in many organs. The spectrum of clinical phenotypes in patients with HNF1B mutations include abnormality of liver, kidney, pancreas and urogenital tract ${ }^{[7,8]}$. There are other common features including delayed language development, learning disability, kidney involvement, and eye dysmorphism and strabismus ${ }^{[3]}$. The most prevalent findings in patients with $17 \mathrm{q} 12$ deletion were kidney abnormalities. This case had not the most common kidney abnormality, i.e. renal cysts, renal tubule dysfunction was her highlighted clinical manifestation. HNF1B recognizes a site containing ion transport regulator 2 gene, which can cause hypomagnesaemia with hypermagnesuria and hypocalciuria ${ }^{[9]}$. Motyka had reported four cases of MODY5. These patients all had various degrees of hypomagnesaemia ${ }^{[10]}$. $74 \%$ of MODY5 patients had renal insufficiency with hypomagnesaemia and hypocalcinuria. This is related to renal abnormalities associated with MODY5. This patient suffered refractory hypomagnesaemia to further identify the cause and was proved to exist deletion of HNF1B gene.

The HNF1B gene also plays an important role in the development of the pancreas. Many patients with $17 \mathrm{q} 12$ recurrent deletion syndrome have pancreatic atrophy and dysplasia. Pancreatic anomalies were difficult to detect by ultrasound, CT or magnetic resonance imaging should be performed. There was no pancreas abnormality in our case by ultrasound examination and CT examination was not performed.

There is no precise epidemiological data on $17 \mathrm{q} 12$ recurrent deletion syndrome. The prevalence of $17 \mathrm{q} 12$ recurrent deletion syndrome in Denmark was about 1.6 per 1,000,000 citizens $^{[11]}$. The morbidity of $17 \mathrm{q} 12$ recurrent deletion syndrome in the Chinese population is unclear. But there are some case reports of $17 \mathrm{q} 12$ recurrent deletion syndrome. It was usually reported in fetuses with congenital renal abnormalities ${ }^{[12]}$.

Neurological and psychiatric problems were common manifestations in patients with $17 \mathrm{q} 12$ deletion. This patient had no such symptoms. She showed normal intellectual development and no abnormal mental behavior. On account of absence of structural abnormalities of the genitourinary system and neurological manifestation, this patient was likely to be diagnosed with single MODY5.

Elevated liver enzymes were reported in $40 \%$ of individuals with $17 \mathrm{q} 12$ recurrent deletion syndrome ${ }^{[11,13]}$. Live cysts, hepatomegaly, cholestasis, and steatohepatitis were also previously reported $^{[11,14]}$. This case had no elevated liver enzymes and morphological abnormalities. Calcification plaque was found in her liver. Gastrointestinal diseases were less common features. Gastroesophageal reflux disease, duodenal atresia and hiatus hernia were reported previously ${ }^{[11,13,15]}$. Chronic non-atrophic gastritis with bile reflux was revealed under gastroscopy in this patient. About one third of females and a quarter of males with $17 \mathrm{q} 12$ 
recurrent deletion syndrome have genital abnormalities. However, genital abnormalities were not found in this patient.

Compared to the patients with point mutations in HNF1B, the patients of 17q12 recurrent deletion syndrome have earlier onset age, more symptoms and lower $\mathrm{BMI}^{[16]} .17 \mathrm{q} 12$ recurrent deletion syndrome may also co-exist with other genetic abnormalities. Cohen and colleagues have reported a monozygotic twin patient with Williams syndrome ${ }^{[17]}$. In addition to the typical deletion at $7 \mathrm{q} 11.23,1.7 \mathrm{Mb}$ deletion in the $17 \mathrm{q} 12$ region was found on microarray comparative genomic hybridization ${ }^{[17]}$.

The phenotype of $17 \mathrm{q} 12$ recurrent deletion syndrome is variable due to the differences of genotype ${ }^{[6]}$. The variation in severity of the disease makes the necessity for close surveillance of deletion carriers. It is regrettable that other relatives of this patient refused to accept relevant examinations to identify the presence of $17 \mathrm{q} 12$ deletion syndrome.

Because this disease is rare and genetic testing is expensive, the HNF1B score has been adopted to screen for suspicious patients. The HNF1B score is a simple tool to select patients for HNF1B gene analysis, but more research is needed to confirm the cutoff point to improve diagnostic accuracy ${ }^{[18,19]}$.

Whether $17 \mathrm{q} 12$ recurrent deletion syndrome existed or not, insulin is the main treatment for MODY5 on account of decreased islet function ${ }^{[20]}$. Although this patient was administered oral hypoglycemic drugs in the early stage, diabetic ketoacidosis manifested soon due to the severe deficiency of islet function. Therefore, insulin therapy was necessary.

\section{References}

[1] Nkonge KM, Nkonge DK, Nkonge TN. The epidemiology, molecular pathogenesis, diagnosis, and treatment of maturity-onset diabetes of the young (MODY). Clin Diabetes Endocrinol. 2020, 6(1):20. doi: 10.1186/s40842-020-00112-5.

[2] Yalçın Çapan Ö, Aydın N, Yılmaz T, et al. Whole exome sequencing reveals novel candidate gene variants for MODY. Clin Chim Acta. 2020, 510:97-104. doi: 10.1016/j.cca.2020.07.005.

[3] Shields BM, Hicks S, Shepherd MH, et al. Maturity-onset diabetes of the young (MODY): how many cases are we missing? Diabetologia. 2010, 53(12):2504-2508. doi: 10.1007/s00125-010-1799-4.

[4] Jang KM. Maturity-onset diabetes of the young: update and perspectives on diagnosis and treatment. Yeungnam Univ J Med. 2020, 37(1):13-21. doi: 10.12701/yujm.2019.00409

[5] Peixoto-Barbosa R, Reis AF, Giuffrida FMA. Update on clinical screening of maturity-onset diabetes of the young (MODY). Diabetol Metab Syndr. 2020, 12:50. doi: 10.1186/s13098-020-00557-9.

[6] George AM, Love DR, Hayes I, et al. Recurrent transmission of a 17q12 microdeletion and a variable clinical spectrum. Mol Syndromol. 2012, 2(2):72-75. doi: 10.1159/000335344.

[7] Mateus JC, Rivera C, O'Meara M, et al. Maturity-onset diabetes of the young type 5 a MULTISYSTEMIC disease: a CASE report of a novel mutation in the HNF1B gene and literature review. Clin Diabetes Endocrinol, 2020, 6:16. doi: 10.1186/s40842-020-00103-6.

[8] Sztromwasser P, Michalak A, Małachowska B, et al. A cross-sectional study of patients referred for HNF1B-MODY genetic testing due to cystic kidneys and diabetes. Pediatr Diabetes, 2020, 21(3):422-430. doi: 10.1111/pedi.12959. 
[9] Heidet L, Decramer S, Pawtowski A, et al. Spectrum of HNF1B mutations in a large cohort of patients who harbor renal diseases. Clin J Am Soc Nephrol 2010, 5(6):1079-1090. doi: 10.2215/CJN.06810909.

[10] Motyka R, Kołbuc M, Wierzchołowski W, et al. Four cases of maturity onset diabetes of the young (MODY) type 5 associated with mutations in the hepatocyte nuclear factor 1 beta (HNF1B) gene presenting in a 13-year-old boy and in adult men aged 33, 34, and 35 years in Poland. Am J Case Rep. 2021, 22:e928994. doi: 10.12659/AJCR.928994.

[11] Rasmussen M, Vestergaard EM, Graakjaer J, et al. 17q12 Deletion and duplication syndrome in Denmark - a clinical cohort of 38 patients and review of the literature. Am J Med Genet A. 2016, 170(11) :2934-2942. doi: 10.1002/ajmg.a.37848.

[12] Wan SN, Zheng YY, Dang YH, et al. Prenatal diagnosis of 17q12 microdeletion and microduplication syndrome in fetuses with congenital renal abnormalities. Mol Cytogenet. 2019, 12:19. doi: 10.1186/s13039-019-0431-7.

[13] Quintero-Rivera F, Woo JS, Bomberg EM, et al. Duodenal atresia in 17q12 microdeletion including HNF1B: a new associated malformation in this syndrome. Am J Med Genet A. 2014, 164A:3076-3082. doi: 10.1002/ajmg.a.36767.

[14] Raile K, Klopocki E, Holder M, et al. Expanded clinical spectrum in hepatocyte nuclear factor 1B-maturity-onset diabetes of the young. J Clin Endocrinol Metab. 2009, 94(7):2658-2664. doi: 10.1210/jc.2008-2189.

[15] Goumy C, Laffargue F, Eymard-Pierre E, et al. Congenital diaphragmatic hernia may be associated with 17q12 microdeletion syndrome. Am J Med Genet A. 2015, 167A(1):250-253. doi: 10.1002/ajmg.a.36840.

[16] Wu HX, Li L, Zhang H, et al. Accurate diagnosis and heterogeneity analysis of a 17q12 deletion syndrome family with adulthood diabetes onset and complex clinical phenotypes. Endocrine. 2021, 73(1):37-46. doi: 10.1007/s12020-021-02682-5.

[17] Cohena L, Samanicha J, Panb Q, et al. 17q12 Deletion in a patient with Williams syndrome: Case report and review of the literature. Journal of Pediatric Genetics. 2012, 1(2):135-141. doi: 10.3233/PGE-2012-022.

[18] Faguer S, Chassaing N, Bandin F, et al. The HNF1B score is a simple tool to select patients for HNF1B gene analysis. Kidney Int. 2014, 86(5):1007-1015. doi: 10.1038/ki.2014.202.

[19] Clissold R, Shields B, Ellard S, et al. Assessment of the HNF1B score as a tool to select patients for HNF1B genetic testing. Nephron. 2015, 130(2):134-140. doi: $10.1159 / 000398819$.

[20] Delvecchio M, Pastore C, Giordano P. Treatment options for MODY patients: a systematic review of literature. Diabetes Ther. 2020, 11(8):1667-1685. doi: 10.1007/s13300-020-00864-4. 